\title{
Different Thought Patterns and Chinese College English Writing
}

\author{
REN Rong \\ English Department, \\ Yuanpei College of Shaoxing University, \\ Shaoxing, Zhejiang, China
}

\begin{abstract}
People are used to attributing the less-proficient Chinese College English writing to students' inaccuracy of diction, spelling, and grammar, while paying little attention to the impact of different thought patterns beneath their words. This paper holds that different thought pattern is one of the striking factors which influence Chinese College English writing. The paper analyses the cultural roots of the different thought patterns, ranging from the impacts of the different word system, philosophical attitudes to Baguwen, the Chinese writing genre in feudal society, and the west theology. As a result, the westerners tend to think in a way of logic, analysis and dichotomy while Chinese in a way of intuition, synthesis and unity, which causes the writing redundant and spiraling in terms of structure, style and cohesion. In the end, the author appeals for the Chinese College English teachers to cultivate a keen awareness of the different thought patterns among different writers. Only by bewaring of different thought patterns can we further improve Chinese College English writing ability.
\end{abstract}

Key words- thought patterns; College English writing; difference

\section{INTRODUCTION}

Chinese College English teachers have traditionally laid too much emphasis on sentence accuracy such as diction, spelling, grammaticality in the writing course, while paying little attention to the teaching of organization and arrangement of the whole passage. As a result, the students may produce grammatically correct writings with the paragraphs redundant, topic and developing sentences lacking, and what's worse, with the theme departed. In other words, they cannot write English in an English way in terms of textual cohesion. Different scholars (R. B. Kaplan, 1996; Wang Moxi and Li Jin, 1993; Yu Qiuling, 2001) have listed quite a few reasons for the incompetence of Chinese writing, and my years of experience in teaching English writing brings me into a complete reconciliation with one of the explanations that: different thought pattern is the striking factor which influences Chinese college students' English writing.

A. A contrastive analysis of English and Chinese thought patterns

Thought pattern refers to the habit or the way people tend to think. It is a structure or process by which people cognize, understand, and value the objective reality. Different cultures have different ways of thinking. Different thought patterns result in different textual organizational structure (Jia Yuxin, 1997). It is generally believed that the thought pattern of people in the west is linearly, which is characterized as follows: people first summarize what they want to express into a topic sentence or a thesis statement in writing, then illustrate the main idea with examples or details. Differently, the thought pattern of people in the east, typically Chinese, is spiraling. They are likely to narrate by indirection (Kaplan, 1996). Chinese people are used to wandering about the topic, without extending the topic directly to the point. Even though some scholars disagree with Kaplan's theoretical hypothesis, it is universally true that every nation has its own inclination to think.

\section{B. The cultural roots of the different thought patterns}

Different thought patterns are culturally rooted. "Culture is really an integral part of the interaction between language and thought. Cultural patterns, customs, and ways of life are expressed in language; culture-specific world views are reflected in language” (Douglas, 1980, P. 141). The causes for the different thought patterns may be as follows:

2.1 Different word system leads to different conceptualization of the world

People in collective culture tend to think in intuitive way, while people in individualistic culture tend to think in a way of reasoning and making a decision, that is, in a logical way (Ji Xianlin, 1991). English and Chinese people are influenced by the above different cultures respectively. The intuitive process of cognitions is particularly the case with Chinese logographs. We may say that Chinese characters are the union of form, meaning, and pronunciation, while English is a kind of alphabetic language, and its word form does not show any relation with its corresponding meaning. As far as grammar is concerned, Chinese people emphasize on the paratactic relation between words; while English, on the hypotactic relations. Grammar is the result of the abstraction of the human thought, so the different word system helps to demonstrate that Chinese people are more capable of specific intuition, while the western people are more competent in logical abstraction. Specific intuition and logical abstraction cause, in turn, inducing and deducing thought patterns in writing.

2.2 The ancient Chinese genre --- Baguwen (eight-legged essay) influences Chinese College English writing 
China is a country with a long history. The long feudal society has formed a set of rigid examination system keju. One of its realizations is Baguwen, which is made up of a stereotyped formula, that is, the writer must abide by eight steps in writing, of which the conclusion must be given at the last part. Under such influences, Chinese students usually do not tell the reader what they want to express until the last part, or they write in climaxing order, making English readers very puzzled. "Well, what's on your mind?" or "Please get down to business." are typical American reactions toward Chinese writers (Jia Yuxin, 1997).

2.3 Different philosophical attitudes have molded different thought patterns

Besides the intuition and logic, other differences between thought patterns are synthesis and analysis, unity and dichotomy. Some scholars (such as Hu Wenzhong and Gao Yihong, 1997, P. 153) hold that the thought pattern of people in the east is synthesis, while the thought pattern of people in the west is analysis. Chinese people have a tendency to think in a synthetic way about things, taking all aspects of things into synthetic consideration before drawing a conclusion. The different thought patterns can be shown between traditional Chinese medicine and the western medicine. Chinese doctors will take into an integral view of the patient's conditions after employing sufficient diagnosis before they prescribe multi-tasting herbs with a synthetic and curative effect for disease. However, the western doctors usually treat the patient's painful part only because they consider the human body as one made up of smaller, independent parts as a result of analytical thinking. Similarly, Chinese sages observe the oneness between Heaven and Man, Yin and Yang. The belief of unity, which derives from "oneness" and "harmony", is typical Chinese way of thinking. Meanwhile, the westerners have a particular tendency to see the world in terms of dichotomy as compared with Chinese harmony. This characteristic is illustrated by the individualistic tendency to think in terms good---evil, right---wrong, yes---no, true---false, beautiful---ugly, freedom---discipline, success---failure... That's one of the reasons why English argumentation is usually very clear in its stand or viewpoint, while Chinese writing is often viewed as indirect, ambiguous, and mysterious.

2.4 The west theology has a deep impact upon the westerners' thought pattern

The middle-aged European writing remains a stereotyped formula, which is typical in Saint Thomas Aquinas, the Italian representative of scholasticism. Under such formula, the author first states the premise before he lists several opposing views against the premise. At last, the author may quote and list many evidences to refute the opposing side so as to support and prove the premise. The underlying thought pattern beneath the formula is something like syllogism, which is closely associated with
English writing and is a result of the logical, analytical and dichotomous thinking. The contemporary English writing patterns, especially in argumentation, fall into this category.

However, the above-mentioned tendencies of the thought patterns are relative rather than absolute. Cultural diversity does not mean that people in individualistic culture think only by logic, analysis, and dichotomy, nor do people in collectivistic cultures think only by intuition, synthesis and unity. The roots involved are the relative emphasis on particular methods of thinking rather than absolute differences.

\section{Thought patterns' negative impact on Chinese College English writing}

Writing is the reflection of one's thought towards the world. Thought pattern plays an important role in any writing. Influenced by the above-mentioned thought patterns, Chinese college students always transfer their thought patterns into English writing, which exerts three-aspect influences as follows:

3.1 The negative impact on the structure of the College English writing

Directed by the Chinese thought pattern, Chinese students are not experienced in organizing an English paragraph. "Errors often occurred as a result of the negative transfer or mother tongue patterns into the learner's L2" (Rod Ellis, 1999, P. 301-302). Here is an example from Wang Moxi's (1993, P. 63) discovery after his scientific investigation (some mistakes are not revised).

\section{WHY IS ENGLISH IMPORTANT TO SCIENTISTS}

We live in a world which science and technology is developing very quickly. The developing of science needs scientists from all over the world exchanges their work more and more frequently. However, the scientist come from different countries usually speak different languages. If there isn't a common language that can be understood by everybody, they won't understand each other. On the other hand, we wish to know what others are doing and what experiences and what knowledge and things we should learn to improve our work, so we must master the foreign language. However, there are countless languages in the world, we can't learn all of them. Fortunately, there is one kind of language that is common used all over the world, English. Most of the scientists of the world can speak and read and write English, most of the important academic janorals are in English, and all the international academic meeting use English, we can go to any corner of the world to exchange with the people without know their mother language. We can say we are holding one of the very powerful weapons of study science. (Wang Moxi, 1993, P. 63)

Written by a Chinese college student, this paragraph is the typical product of the Chinese thought pattern. At the 
beginning of the paragraph, the author wants to express the importance of English, but he does not develop it directly and forcefully from the beginning to the end. With the help of such connectors as "necessary", "must ", "want”, "have to", "if not", the author does not achieve to defend the topic. The whole paragraph lacks a clear topic sentence, which is usually indispensable in native speaker's writing. The structure of the paragraph is not problem-solution pattern, nor general-particular pattern, nor matching pattern, which are typical English writing patterns. Since the topic is implied in the paragraph, we may categorize such Chinese writing into the "implied pattern".

3.2 The negative impact on the style of the College English writing

The style of modern English is characterized by its briefness, forcefulness, and simplicity, but seldom is the Chinese College English writing style so. Influenced by the negative transfer of the Chinese thought pattern, they usually jam a sentence with too many allusions, metaphors, idioms and other jargons, therefore, making the English sentence redundant, superfluous, and wordy. Here are some examples:

1. Weak: ... in spite of some disadvantages, advertising is very essential to all of us in modern life. (Mao Ronggui, 1997, P. 128)

Improved: ...in spite of some disadvantages, advertising is essential to modern life.

2. Weak: The bag was as heavy as a mountain.

Improved: The bag was very heavy.

3. Weak: Last night he was suddenly struck by an idea: no pains, no gains.

Improved: Last night he was suddenly struck by an idea: Bashful knight never won fair maiden. (Mao Ronggui, 1997, P. 473)

Chinese students frequently employ sayings such as "Time flies like an arrow", "No pains, no gains", "By reviewing old knowledge one can acquire new", but such sayings are viewed as "clichés" to native speakers. "In U.S. culture we place far greater value of the work of the writer who expresses ideas in fresh, new ways than on the one who staggers about on the crutch of cliché use" (Mao Ronggui, 1997, P. 471). The above mentioned style problem does not have no relation with the different thought patterns on sayings.

3.3 The negative impact on the cohesion of College English writing

Good English writing must be coherent, but college students seem to be poor in connecting sentences. We may attribute this to the different grammatical characteristics between English and Chinese. English grammar is explicit, while Chinese grammar is implicit; English emphasizes on hypotactic relations, while Chinese emphasizes on paratactic relations. The different word systems reflect different thought patterns, which in turn, reflect different textual connecting methods. Even though there are connectors in Chinese, too, Chinese students are not likely to use or even not use such cohesive devices. The following sentences are cases in point:

1. Faulty: We should not waste our time on playing games; we should spend time on studies.

Revised: Instead of wasting it on playing games, we should spend time on studies.

2. Faulty: Pollution has become a serious problem; we should take steps to control it.

Revised: Since pollution has become a serious problem, we should take steps to control it.

3. Weak: I got up early last Monday. I had my breakfast in a hurry. At seven o'clock I arrived at the school. I was going to take part in the English Speaking Contest.

Improved: I got up early last Monday, because I was going to take part in the English Speaking Contest. I had my breakfast hurriedly and at seven o'clock, I arrived at the school.

As can be seen, it is no difficult to find Chinese thought traces in the above examples.

\section{CONCLUSION}

Different thought patterns interfere in English and Chinese writing. They are rooted in different culture, or different philosophy, word system, and history in specific. Greatly influenced by the negative transfer of the Chinese thought pattern, Chinese college students are inefficient in English writing as far as the structure, style, and cohesive devices are concerned. On this basis, we must spare no effort to adapt the Chinese mind to the English ways of thinking. We should not spend too much time on merely guiding the students to be keenly aware of the syntactical accuracy, or grammatical appropriateness, but spend time on training them to be competent in organizing the structure, enhancing the coherence, avoiding the negative transfer of the Chinese thought pattern to the full extent in English writing, and cultivating the students' cultural awareness at last. Only in this way can we further improve our students' College English writing ability.

\section{REFERENCES}

[1] Douglas, B. H. 1980. Principles of Language Learning and Teaching. New Jersey: Prentice-Hall, Inc.

[2] Ellis, R. 1999. The Study of Second Language Acquisition. Shanghai: Shanghai Foreign Language Education Press.

[3] He, Mingzhu. 2000. "Writing English the English way------ A study of the causes of Chinglish”, Teaching English in China, 4: 25-28.

[4] Ji, Xianlin. 1991. “Zai lun dongfang wenhua”, Quan Yan, 5.

[5] Jia, Yuxin. 1997. Intercultural Communication. Shanghai: Shanghai Foreign Language Education Press.

[6] Kaplan, R. B. 1996. "Cultural thought patterns in intercultural education”, Language Learning: A Journal of Applied Linguistics, XVI: $1-2$.

[7] Mao, Ronggui. 1997. Zhonguo Daxuesheng Zuowen Pinggai. Shanghai: Shanghai Foreign Language Education Press. 
[8] Tao, Jiawei. 1998. Writing and Culture. Shanghai: Shanghai Foreign Language Education Press.

[9] Wang, Moxi, \& Li, Jin. 1993. "Zhongguo xuesheng yingyu yupian siwei moshi diaocha”, Foreign Language Teaching and Research, 4: 63.

[10] Wu, Wenzhong \& Gao, Yihong. 1997. Foreign Language Education and Culture. Changsha: Hunan Education Press.

[11] Yu, Qiuling. 2001. "Yinghan duibi xiuci yanjiu yu zuowen jiaoxue", Fujian Foreign Language, 2: 33-36. 